\title{
Mayo Clinic Cancer Center
}

National Cancer Institute

\section{Source}

National Cancer Institute. Mayo Clinic Cancer Center. NCI Thesaurus. Code C39454.

The Mayo Clinic Cancer Center is dedicated to understanding the biology of cancer, to discovering new ways to predict, prevent, diagnose and treat cancer, and to transforming the quality of life for cancer patients today and in the future. Mayo's philosophy of patient care is that medicine is best practiced as a cooperative science with clinicians, specialists, and researchers working as a team. It became an $\mathrm{NCl}$-designated Comprehensive Cancer Center in 1971. 